\title{
BMJ Open Can we Save the rectum by watchful waiting or TransAnal microsurgery following (chemo) Radiotherapy versus Total mesorectal excision for early REctal Cancer (STAR-TREC study)?: protocol for a multicentre, randomised feasibility study
}

\author{
Anouk J M Rombouts, ${ }^{1}$ Issam Al-Najami, ${ }^{2}$ Natalie L Abbott, ${ }^{3}$ Ane Appelt, ${ }^{4,5}$ \\ Gunnar Baatrup, ${ }^{2}$ Simon Bach, ${ }^{6}$ Aneel Bhangu, ${ }^{6}$ Karen-Lise Garm Spindler, ${ }^{7,8}$ \\ Richard Gray, ${ }^{9}$ Kelly Handley, ${ }^{10}$ Manjinder Kaur, ${ }^{11}$ Ellen Kerkhof, ${ }^{12}$ \\ Camilla Jensenius Kronborg, ${ }^{13}$ Laura Magill, ${ }^{11}$ Corrie A M Marijnen, ${ }^{12}$ \\ Iris D Nagtegaal, ${ }^{14}$ Lars Nyvang, ${ }^{15}$ Femke P Peters, ${ }^{12}$ Per Pfeiffer, ${ }^{16}$ Cornelis Punt, ${ }^{17}$ \\ Philip Quirke, ${ }^{18}$ David Sebag-Montefiore, ${ }^{19}$ Mark Teo, ${ }^{19,20}$ Nick West, ${ }^{18}$ \\ Johannes H W de Wilt, ${ }^{1}$ for STAR-TREC Collaborative Group
}

To cite: STAR-TREC Collaborative Group. Can we Save the rectum by watchful waiting or TransAnal microsurgery following (chemo) Radiotherapy versus Total mesorectal excision for early REctal Cancer (STARTREC study)?: protocol for a multicentre, randomised feasibility study. BMJ Open 2017;7:e019474. doi:10.1136/ bmjopen-2017-019474

- Prepublication history for this paper is available online. To view these files, please visit the journal online (http://dx.doi. org/10.1136/bmjopen-2017019474).

Received 25 September 2017 Accepted 20 October 2017

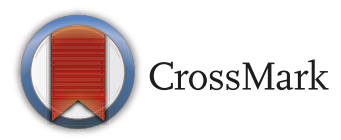

For numbered affiliations see end of article.

Correspondence to

Professor Johannes H W de Wilt; star-trec@radboudumc.nl

\section{ABSTRACT}

Introduction Total mesorectal excision (TME) is the highly effective standard treatment for rectal cancer but is associated with significant morbidity and may be overtreatment for low-risk cancers. This study is designed to determine the feasibility of international recruitment in a study comparing organ-saving approaches versus standard TME surgery.

Methods and analysis STAR-TREC trial is a multicentre international randomised, three-arm parallel, phase II feasibility study in patients with

biopsy-proven adenocarcinoma of the rectum. The trial is coordinated from Birmingham, UK with national hubs in Radboudumc (the Netherlands) and Odense University Hospital Svendborg UMC (Denmark). Patients with rectal cancer, staged by CT and MRI as $\leq \mathrm{CT} 3 \mathrm{~b}$ (up to $5 \mathrm{~mm}$ of extramural spread) NO MO can be included. Patients will be randomised to either standard TME surgery (control), organ-saving treatment using long-course concurrent chemoradiation or organ-saving treatment using short-course radiotherapy. For patients treated with an organ-saving strategy, clinical response to (chemo) radiotherapy determines the next treatment step. An active surveillance regime will be performed in the case of a complete clinical regression. In the case of incomplete clinical regression, patients will proceed to local excision using an optimised platform such as transanal endoscopic microsurgery or other transanal techniques (eg, transanal endoscopic operation or transanal minimally invasive surgery). The primary endpoint of this phase II study is to demonstrate sufficient international recruitment in order to sustain a phase III study incorporating pelvic failure as the primary endpoint. Success in phase II is
Strengths and limitations of this study

- This phase II study is the first study to randomise between the standard of care in early rectal cancer (ie, total mesorectal excision surgery) and two organ-saving strategies using (chemo)radiotherapy followed by selective transanal microsurgery.

- STAR-TREC study will show whether it is feasible to recruit enough patients for a consecutive international large, multicentre randomised phase III trial.

- The study design incorporates several adjustments in standard (chemo)radiation therapy protocols intended to reduce treatment-related side effects associated with organ-saving therapy.

- Clinical nodal staging of rectal cancer is rather unreliable and patients with false-negative nodal disease will be included in the study.

- Experience with clinical judgement of a complete response is difficult and needs to be monitored carefully with central reviewing during the study.

defined as randomisation of at least four cases per month internationally in year 1 , rising to at least six cases per month internationally during year 2 .

Ethics and dissemination The medical ethical committees of all the participating countries have approved the study protocol. Results of the primary and secondary endpoints will be submitted for publication in peer-reviewed journals.

Trial registration number ISRCTN14240288, 20 0ctober 2016. NCT02945566; Pre-results, October 2016. 


\section{INTRODUCTION}

The introduction of bowel cancer screening is associated with a significant increase in the incidence of earlystage rectal cancer. ${ }^{12}$ Total mesorectal excision (TME) surgery is an effective oncological treatment for earlystage rectal cancer, only $2 \%$ and $12 \%$ of patients experience local or distant failure, respectively. ${ }^{3-5}$ However, standard surgery for rectal cancer requires permanent stoma formation in 10\%-20\% of cases and temporary stoma formation in $60 \%-70 \% .{ }^{67}$ Many temporary stomas are not reversed. ${ }^{89}$ Furthermore, TME surgery is associated with major morbidity and mortality in a significant number of patients. Over $50 \%$ of all patients following TME surgery experience faecal incontinence, whereas urinary problems and sexual dysfunction are observed in $32 \%-80 \%$ of patients. ${ }^{10-14}$ Another complication following TME surgery is anastomotic bowel leakage which occurs in approximately $15 \%$ of patients. ${ }^{15}$ In addition, quality-of-life studies show that TME surgery is associated with persistently poor social role and body image. ${ }^{12}$ 16-19 Mortality following TME surgery rises with age; the 6-month mortality following TME surgery is $2.0 \%-4.6 \%$ for young patients with rectal cancer and $9.0 \%-13.4 \%$ for elderly patients (aged $>75$ years).$^{20-22}$ There are concerns that TME surgery, which evolved to treat locally advanced, symptomatic tumours, may result in significant overtreatment of early screen-detected tumours. An organ-preserving strategy may generate significantly less morbidity without substantially compromising oncological outcomes. Promising outcomes have been reported for (chemo)radiation therapy followed by watchful waiting or local excision.

Habr-Gama's group have notably published a watchful waiting approach to rectal cancer. Of 265 patients with predominantly T3 rectal cancer treated with chemoradiation therapy (CRT), 71 patients $(27 \%)$ had a complete clinical response (cCR). ${ }^{2324}$ These patients did not have surgery and after a mean follow-up of 57 months (range: 18-156), only two patients developed local recurrence, one of which was successfully salvaged. A Dutch group then prospectively selected patients with cCR for a watchful waiting strategy $(n=21) .^{25}$ After a mean follow-up 25 months $( \pm 19$ months $)$, one patient had developed a local recurrence which was salvaged by surgery and all other patients were alive without disease. In 2015, the effect of a radiation boost after CRT was evaluated in a prospective observational Danish study. A watch and wait policy was possible in 40 out of 51 included patients. ${ }^{26}$ At 1 year, local recurrence occurred in $16 \%$ of 40 patients who initially showed a cCR. Rectal bleeding was relatively frequent in this study during follow-up perhaps relating to the higher radiotherapy doses that were used. However, these results which combine high cCR rates and low local recurrence rates have not been consistently replicated. ${ }^{27} 28$ Furthermore, CRT is associated with treatment-related morbidity and a mortality rate of $0.5 \%-1 \%$ should be considered. ${ }^{29}$

Another organ-saving treatment strategy is local excision instead of radical surgery. Early rectal tumours may be locally excised through the anus with low morbidity and mortality using transanal endoscopic microsurgery (TEM), allowing rectal-saving treatment. ${ }^{30}$ Morbidity and mortality after local excision are much lower than after major resection. Morbidity associated with TEM includes bowel perforation, (transitory) incontinence, wound infection and local pain. ${ }^{32-34}$ In a study of 5305 patients with early-stage rectal cancer, 30-day mortality after local excision was found to be $0.5 \%$ compared with $2.4 \%$ in patients undergoing major resection $(\mathrm{P}=0.008)$. Morbidity within 30 days of surgery was $4.4 \%$ in the local excision group versus $12.7 \%$ in the major resection group $(\mathrm{P}<0.001) .{ }^{34}$ However, the risk of non-radical resection after local excision is higher and the risks of leaving behind microscopic lymph node metastases are a potential cause of local failure. ${ }^{53}$ The incidence of lymph node metastasis ranges from $6 \%$ to $14 \%$ for $\mathrm{T} 1$ tumours, $17 \%$ to $23 \%$ for T2 tumours and $49 \%$ to $66 \%$ for T3 tumours. ${ }^{36}$

Combining radiotherapy with TEM could possibly lead to better outcomes because radiotherapy can effectively treat microscopic mesorectal nodal metastases and contribute to tumour downsizing. ${ }^{37} 38$ However, limited prospective evidence currently exists to guide the use of radiotherapy and local excision as curative treatment for early rectal cancer. Lezoche et al randomised 100 patients with T2N0 rectal cancer to CRTfollowed by laparoscopic TME surgery or CRT and TEM with a $6-8$ week interval to surgery. ${ }^{39}$ After a median follow-up of 9 years, local recurrence rates were $6 \%$ and $8 \%$ in the TME and TEM arms, respectively. In a trial of 89 patients with unfavourable cT1N0, cT2N0 or borderline cT2/3N0 tumours by Bujko et al, patients were given neoadjuvant treatment with short-course radiation therapy (SCRT) or CRT prior to delayed local excision. ${ }^{40}$ No further treatment was offered for good responders, whereas immediate TME surgery was recommended for all other patients. Good responders had a 2-year local recurrence rate of $10 \%$. Of the poor responders, eight patients had a TME and none of this group had a recurrence, however, 18 declined or were unfit for TME, and this group had a 2-year local recurrence rate of $37 \%$. This underlines that in high-risk or poor responding patients, neoadjuvant radiotherapy followed by local excision is inadequate treatment.

A study investigating chemoradiation therapy for rectal cancer in the distal rectum followed by organ-sparing transanal endoscopic microsurgery (CARTS) was a non-randomised phase II study that evaluated CRT followed by TEM in 55 patients with stage T1-3N0 rectal cancer. ${ }^{41}$ Clinical response was assessed 6-8 weeks after completion of CRT and TEM was performed. Organ saving was achieved in more than half of patients and 21 had ypT0 disease. Radiotherapy consisted of $50 \mathrm{~Gy}$ in 25 fractions and capecitabine $825 \mathrm{mg} / \mathrm{m}^{2}$ two times per day was given for the same period 7 days per week. However, $42 \%$ of patients developed at least grade 3 toxicity and there were two toxicity-related deaths. A multicentre cohort study from the UK that employed SCRT with TEM after 10 weeks demonstrated that $43 / 62$ cases had either no or 
minimal residual disease following radiotherapy. ${ }^{42}$ None of these patients experienced short-term pelvic relapse and treatment-related toxicity was low. The ACOSOG Z6041 study, a single-arm phase II study, evaluated an oxaliplatin and capecitabine concurrent chemotherapy schedule combined with 54 Gy of pelvic radiotherapy followed by TEM for T2N0 rectal cancer. ${ }^{43}$ Both radiotherapy and chemotherapy schedules required reduction during the study due to acute toxicity. Only 3 out of 79 evaluable patients experienced local failure as first event. TREC is a phase II UK study evaluating the feasibility of randomising patients to receive either organ-saving treatment with SCRT and TEM versus standard TME surgery. This study is due to report in 2017 having completed minimum 2-year follow-up.

In conclusion, several strategies can be followed to improve the quality of life of patients with rectal cancer by aiming for organ preservation. However, all data so far are derived from small phase II studies and many questions regarding the optimal radiotherapy schedule and the optimal timing of evaluation remain. In addition, prospective comparative data with radical surgery are not available. Therefore, there is an urgent need for a randomised phase III trial to establish the risks, complication rates and benefits of organ saving compared with standard radical surgery for early-stage rectal cancer. The aim of STAR-TREC study is to assess the feasibility of successfully recruiting to a large, multicentre randomised trial comparing radical surgery versus organ saving treatment using (chemo)radiotherapy followed by selective transanal microsurgery.

\section{METHODS AND ANALYSIS \\ Design}

STAR-TREC trial is a multicentre international randomised, three-arm parallel study in patients with biopsy-proven adenocarcinoma of the rectum. The trial is coordinated from Birmingham, UK with national hubs in Radboudumc (the Netherlands) and Odense University Hospital Svendborg UMC (Denmark). Participants are currently being recruited and enrolled; the first patient was enrolled in July 2017.

The primary endpoints of STAR-TREC study (phase II) are defined as:

1. Year 1: Randomise at least four cases per month internationally $(n=48)$.

2. Year 2: Randomise at least six cases per month internationally $(\mathrm{n}=72)$.

The secondary endpoints of this phase II trial are:

1. Year 1: Can one international partner procure independent funding in year 1? Successful international collaboration will be necessary to deliver a future phase III study.

2. Year 1: Can one international partner open the study to recruit in year 1 ?

3. Efficacy of organ-preserving treatment arms on completion of phase II study: Is the organ-saving rate
$>50 \%$ at 12 months (following randomisation) in the experimental arms?

Additional outcome measures pertinent to a future phase III study examining the safety and efficacy of organ saving versus standard surgery will also be collected.

\section{Safety}

- accuracy of MRI in predicting STAR-TREC eligibility;

- 30-day and 6-month mortality;

- surgical morbidity;

- rate of tumour recurrence or regrowth within the bowel wall (experimental arm);

- rate of tumour recurrence within the mesorectum (experimental arm);

- rate of distant metastases;

- pelvic failure rate: expressed as a sum of the following: (1) unresectable pelvic tumour, (2) cases requiring beyond TME surgery or (3) tumour recurrence or regrowth $\leq 1 \mathrm{~mm}$ from the circumferential surgical margin after TME surgery. This outcome measure will be pivotal in challenging current clinical practice and it is our intention that it becomes the primary endpoint in phase III;

- bowel, bladder and sexual dysfunction (baseline and 12, 24 months postrandomisation).

\section{Efficacy}

- proportion of patients with/withouta stoma at 30 days and 1 year;

- histopathological assessment of tumour downstaging following radiotherapy according to depth of tumour invasion and the incidence of other high-risk features in comparison to non-irradiated (control) group;

- proportion of patients identified by clinical and MRI assessment as suitable for active monitoring;

- conversion rates from organ saving to radical surgery;

- disease-free survival;

- quality of life (baseline and 12, 24 months postrandomisation);

- overall survival.

\section{Study population}

This is a hospital-based study. Centre eligibility depends on a Radiotherapy Trials Quality Assurance which is a mixture of an approved departmental standard operating procedure and successful contouring of a case using the new principles of mesorectal irradiation. Candidates will generally be identified in the endoscopy suite following referral for: (1) the investigation of new bowel symptoms, (2) as part of a personal bowel surveillance programme or (3) through national bowel screening. Subjects will then be referred on to either a colorectal surgeon or the colorectal cancer multidisciplinary team (MDT) meeting. Eligibility will be confirmed at the MDT meeting. The main inclusion and exclusion criteria for the trial are summarised in table 1. 
Table 1 Inclusion and exclusion criteria

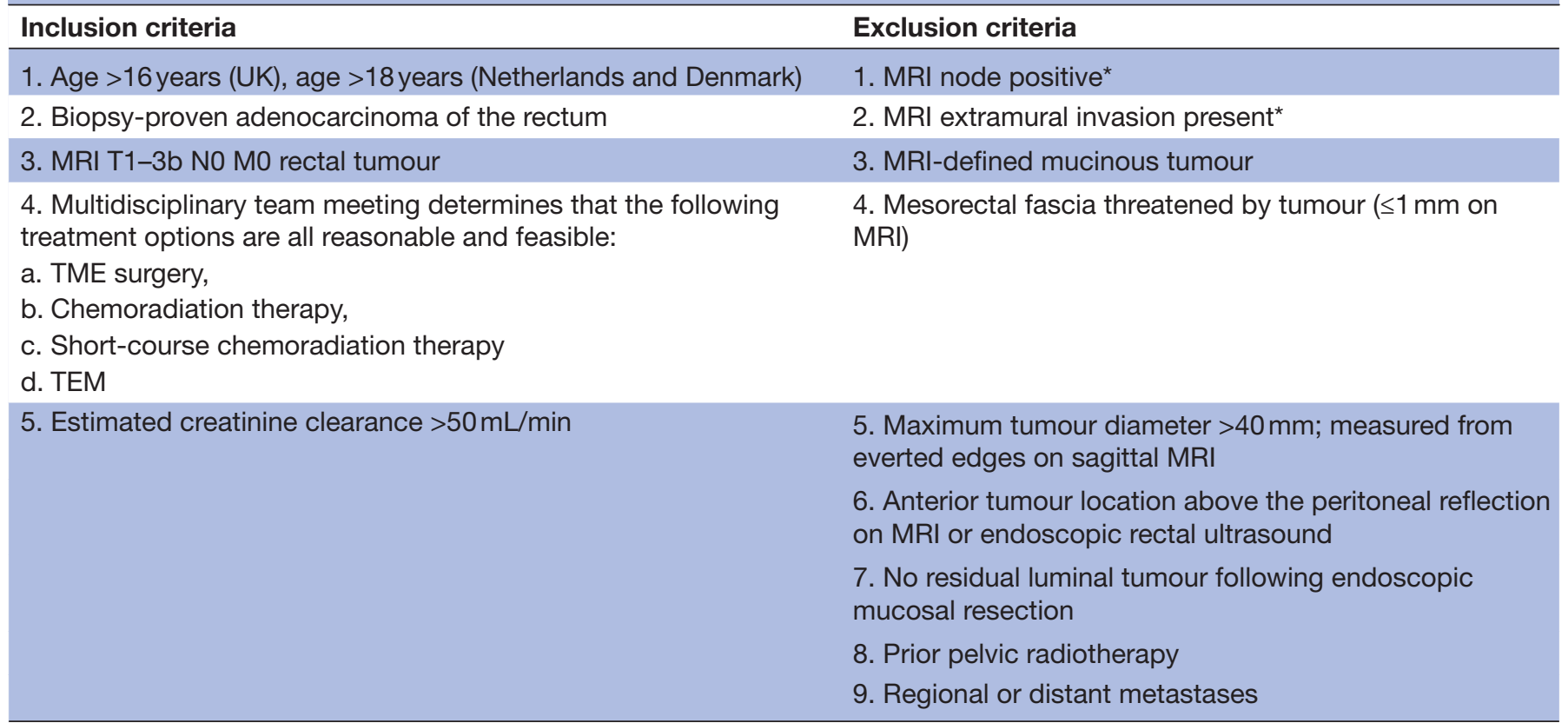

*Defined by protocol guidelines.

TEM, transanal endoscopic microsurgery; TME, total mesorectal excision.

\section{Study arms}

Patients will be randomised to either standard TME surgery (control), organ-saving treatment using longcourse concurrent CRT or organ-saving treatment using SCRT (figure 1). Patients allocated to TME surgery will have a minimum of one abdominal CT scan and regular clinical follow-up will be made according to national guidelines. In the two organ-saving arms, response assessment will take place at 11-13 weeks from the start of (chemo)radiotherapy and again at 16-20 weeks from start. Initial assessment at 11-13 weeks (MRI and endoscopy) will identify a small proportion of cases where radiotherapy has had little or no impact on tumour dimensions. Non-responding patients will be advised to convert to standard TME surgery. Individuals whose tumours demonstrate a satisfactory response at this time point will be examined once again at 16-20 weeks (endoscopy) to determine if a cCR has occurred. It is anticipated that this interval between assessments will allow for additional tumour regression and resolution of acute radiotherapy reactions, facilitating more precise diagnosis of cCR. An active surveillance regime will be performed in the case of a cCR. In the case of incomplete clinical regression, patients will progress to local excision, see figure 1. Representative endoscopic images will be centrally reviewed during this feasibility stage to develop a consistent approach to interpretation of the clinical assessment.

All patients must be assigned to one of the three treatment groups by week 20 :

1. Poor response assessed at 11-13 weeks-patient recommended to convert to radical TME surgery.
2. cCR assessed at 16-20 weeks means that the bowel wall has reverted to normal and patients are treated by watchful waiting.

3. Clinically satisfactory, yet incomplete tumour response at 16-20 weeks, meaning a 50\% or more reduction of tumour size and the presence of any residual mucosal or bowel wall abnormality suggestive of persisting tumour, will prompt local excision by TEM.

\section{Treatment regimen for organ-saving strategies}

Long-course CRT consists of capecitabine and is administered at a dose of $825 \mathrm{mg} / \mathrm{m}^{2}$ two times per day on radiotherapy days only. A total dose of $50 \mathrm{~Gy}$ will be applied to the primary tumour and surrounding mesorectum, in 25 fractions of $2 \mathrm{~Gy}, 5$ days per week.

SCRT consists of a total dose of $25 \mathrm{~Gy}$, applied to the primary tumour and surrounding mesorectum in five fractions of $5 \mathrm{~Gy}$, preferably on five consecutive days. Radiotherapy for organ preservation is primarily aimed at tumour downstaging and can therefore be restricted to the peritumoral area including the primary tumour and the mesorectum resulting in a significant reduction in the irradiated target volume.

\section{Randomisation}

Patients will be randomised on a 1:1:1 basis between standard surgical treatment and organ-saving treatments. Randomisation will be provided by a computer-generated program at the University of Birmingham Clinical Trials Units. The randomisation program will use a minimisation procedure for the following variables:

1. MRI tumour staging ( $\leq \mathrm{T} 3 \mathrm{a} \mathrm{N} 0 \mathrm{~V} 0$ and T3b N0 V0) (T3a: tumour extends $<1 \mathrm{~mm}$ beyond muscularis 


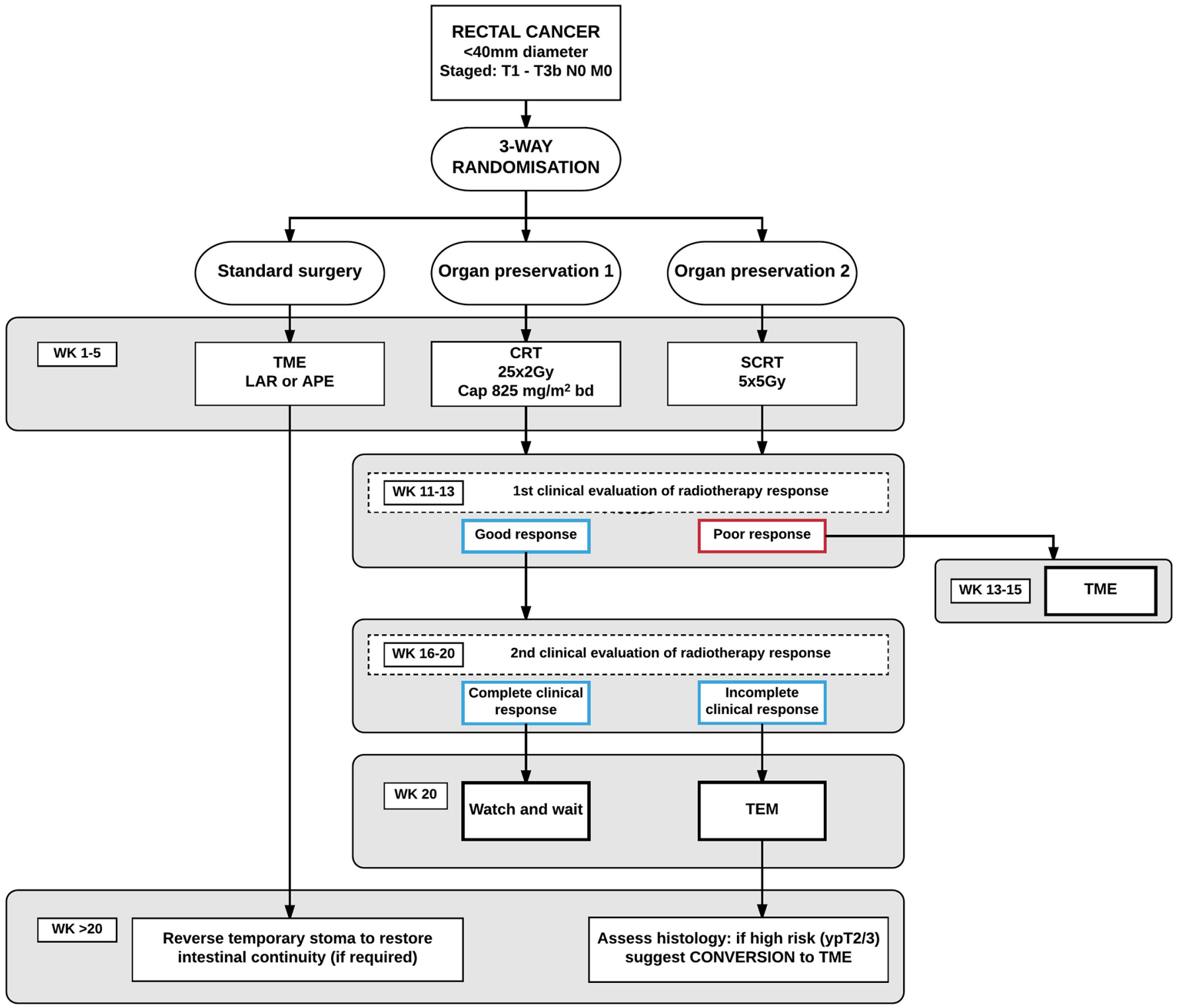

Figure 1 Flow chart of the inclusion, randomisation and management of the study subjects in STAR-TREC trial. APE, anterior perianal excision; CRT, chemoradiation therapy; LAR, low anterior resection; SCRT, short-course radiation therapy; TEM, transanal endoscopic microsurgery; TME, total mesorectal excision.

propria; T3b: tumour extends $1-5 \mathrm{~mm}$ beyond muscularis propria)

2. country (UK, the Netherlands, Denmark).

Stratification and minimisation will be by T-stage to ensure that the more advanced tumours are equally represented across treatments; stratification and by country will be done to account for any bias arising from any possible differences in pretreatment MRI-based staging assessment.

To avoid any possibility of the treatment allocation becoming too predictable, a random factor will be included within the algorithm whereby for a proportion of the allocations true randomisation will be implemented rather than by using the minimisation allocation.

\section{Sample size}

No power calculation is provided as the primary objective is to show feasibility of recruitment. The aim of the present trial is to include four to six patients per month in order to have a high enough randomisation rate to perform a phase III trial. For a phase III trial, the primary outcome would be 3-year pelvic failure. The null hypothesis is that the increase in the rate of pelvic failure at 3 years with organ preservation compared with standard surgery is less than $7 \%$ absolute difference. Prior data indicate that the pelvic recurrence rate in the radical TME group is $2 \%$. If the true recurrence rate for patients in an experimental arm is $9 \%$ then, using $90 \%$ power and alpha $=0.025$ (to account for two treatment comparisons) would require 117 patients per treatment arm. Anticipating a $10 \%$ dropout rate, we would aim to randomise 400 participants. The final decision for a phase III sample size will be taken from information gained during the feasibility study. Data of the phase II trial will be used for the phase III trial. 


\section{Data management}

Case report forms (CRFs) can be entered online at http:/ / www.bctu.bham.ac.uk/STAR-TREC. Authorised staff at sites will require an individual secure login username and password to access this online data entry system. Paper CRFs must be completed, signed/dated and returned to the National STAR-TREC Trial Office by the investigator or an authorised member of the site research team. Data reported on each CRF should be consistent with the source data or the discrepancies should be explained. If information is not known, this must be clearly indicated on the CRF. All missing and ambiguous data will be queried. All sections are to be completed.

Assessment of the health-related quality of life will be done after the patients have completed a series of questionnaires. The questionnaires European Organisation for Research and Treatment of Cancer Quality of life questionnaires for colorectal cancer quality of life questionnaire C30 and CR29 (EORTC QLQL), standardised questionnaire for use as a measure of health outcome (EQ-5D), Low Anterior Resection Syndrome (LARS) score and Patient-completed questionnaire for evaluating male/female lower urinary tract symptoms and impact on quality of life (ICIQ-MLUTS/ICIQFLUTS) will be done at three time points, at baseline prior to treatment and at follow-up 12 and 24 months after the start of treatment.

All trial records must be archived and securely retained for at least 25 years. No documents will be destroyed without prior approval from the Sponsor, via the central STARTREC trial office. On-site monitoring will be carried out as required following a risk assessment and as documented in the monitoring plan for each participating country. Any monitoring activities will be reported to the central STARTREC office and any issues noted will be followed up to resolution. STAR-TREC will also be centrally monitored; however, additional on-site monitoring may occur if triggered. Further information regarding data management is provided in the study protocol.

\section{Ethics and dissemination}

The trial will be performed in accordance with the recommendations guiding physicians in biomedical research involving human subjects, adopted by the 18th World Medical Association General Assembly, Helsinki, Finland and stated in the respective participating countries laws governing human research, and Good Clinical Practice. The medical ethical committees of all the participating countries have approved the study protocol.

A meeting will be held after the end of the study to allow discussion of the main results among the collaborators prior to publication. Results of the primary and secondary endpoints will be submitted for publication in peer-reviewed journals.

\section{DISCUSSION}

The TREC and CARTS groups have combined with colleagues in Denmark to design STAR-TREC study. Phase
II data from TREC and CARTS justifies a randomised comparison of standard radical surgery versus organsaving treatment using either SCRT or CRT with selective use of transanal microsurgery based on a radiotherapy response assessment. Organ preservation is not standard treatment and testing the feasibility is important to determine the scale of randomised trial that can be performed. The phase II STAR-TREC study will evaluate the feasibility of accelerating recruitment to an international three-arm randomised trial.

The published literature supports use of (chemo) radiotherapy and transanal microsurgery as an alternative to major surgery for curative treatment of early rectal cancer. To date, studies have recruited patients who were highly motivated to organ-preserving treatment. Broader patient populations are yet to be evaluated using these organsaving treatments. In addition, the long-term impact of organ-saving treatment after neoadjuvant treatment, on quality of life and, more importantly, oncological outcome is unknown. Therefore, these organ-preserving strategies should ideally be compared with radical TME surgery which represents the current standard of care for patients with rectal cancer. A randomised trial comparing organ-saving treatment with major surgery might be practice changing for the treatment of patients with early rectal cancer.

In addition, while it seems probable that a strategy of organ saving may produce substantial benefits over conventional radical surgery, the optimum organ-saving treatment schedule remains unclear. Phase II studies suggest that SCRT may have the lowest acute toxicity while CRT may achieve the highest cCR rates. Randomisation between these two strategies with the interval calculated from the start of (chemo) radiotherapy will give insight in the possible difference in efficacy in these early cancers. STAR-TREC is, therefore, an international, multicentre, randomised, phase II feasibility study comprising a 1:1:1 randomisation for eligible subjects with early clinically localised rectal cancer.

While published data supports further evaluation of organ saving in patients with early-stage rectal cancer using either SCRT or CRT followed by transanal microsurgery, it has also become clear that not all patients require surgery. Watchful waiting after complete response is being investigated in patients already in need of CRT. ${ }^{24} 2637$ Other studies introduce (chemo) radiation therapy to the treatment regimen in order to facilitate organ preservation. Current techniques use either SCRT (five fractions of $5 \mathrm{~Gy})^{40} 42$ or concurrent fluoropyridine-based CRT (25 fractions of 1.8 or $2 \mathrm{~Gy}){ }^{38-40} 42$ Radiotherapy is routinely followed by TEM, to remove the portion of bowel wall affected by cancer. However, in a significant proportion of patients, there are no signs of residual tumour following radiotherapy. This is termed a cCR. These patients are likely overtreated by routine transanal microsurgery and therefore possibly subjected to unnecessary surgery-related morbidity. Therefore, patients with a cCR might be better served by a watchful waiting approach. ${ }^{44}$ 
STAR-TREC study design incorporates several developmental steps, each intended to further reduce treatment-related side effects associated with organ-preserving therapy:

1. Modification in the capecitabine dose from $825 \mathrm{mg} /$ $\mathrm{m}^{2}$ two times per day for 7 days per week used in CARTS to $825 \mathrm{mg} / \mathrm{m}^{2}$ two times per day for 5 days per week.

2. Use of a smaller radiotherapy volume incorporating only the primary tumour, rectal wall and mesorectum.

3. Use of a two-step clinical response assessment tool following (chemo)radiation so that (1) poor responders are converted to radical TME surgery at the earliest opportunity while (2) good responders are given more time to determine if they reach cCR and may avoid transanal microsurgery.

4. Selective use of transanal microsurgery/TEM for residual mucosal or bowel wall abnormality suggestive of persisting cancer.

5. Objective comparison of the efficacy of CRT versus SCRT with similar intervals between start of radiotherapy and evaluation.

To date, no significant differences are considered for target volume definition for early or advanced rectal cancers. Target volumes contain at least the primary tumour, the mesorectal fat, presacral and internal iliac nodes. ${ }^{45}$ Given that patients in STAR-TREC will be clinically node negative, the necessity of irradiating presacral and iliac nodes is questionable. Even in the case of unexpected nodal involvement, the majority of involved lymph nodes will be peritumoral in the mesorectum, as demonstrated in a series of 121 patients with locally advanced rectal cancer who underwent CRT. ${ }^{46}$ The radiotherapy volume has therefore been reduced to the mesorectal fat only. To ensure safe introduction of this new technique, strict radiotherapy quality assurance is part of the protocol.

STAR-TREC is designed to achieve a recruitment rate that would provide confidence that extension into a phase III trial is achievable. Further applications for funding, ethics approval and a substantial protocol amendment would be required for this transition.

\section{CONCLUSION}

There is an urgent need for a randomised phase III trial to establish the risks and benefits of organ saving compared with standard TME surgery for early-stage rectal cancer. STAR-TREC trial builds on experience gained through the TREC and CARTS phase II studies. STAR-TREC is a multicentre international randomised phase II study designed to assess the feasibility of recruiting six international patients per month in order to facilitate the evaluation of TME surgery versus organ-saving strategy preceded by (chemo)radiation in two different fractionation schedules. The trial aims to improve the rate of patient recruitment compared with earlier studies and will also introduce a mesorectal target volume with quality assurance. The ultimate goal of this phase II feasibility study is to accelerate to a phase III study comparing TME surgery with two organ-saving treatment regimens.

Author affiliations

${ }^{1}$ Department of Surgery, Radboud University Medical Centre, Nijmegen, The Netherlands

${ }^{2}$ Department of Surgery, Odense University Hospital, Odense, Denmark

${ }^{3}$ Radiotheraphy Trials Quality Assurance Group, Velindre Cancer Centre, Cardiff, UK

${ }^{4}$ Leeds Institute of Cancer and Pathology, University of Leeds, Leeds, UK

'Leeds Cancer Centre, St. James' University Hospital, Leeds, UK

${ }^{6}$ Department of Surgery, University Hospitals Birmingham, Birmingham, UK

${ }^{7}$ Department of Clinical Oncology, Aarhus University Hospital, Aarhus, Denmark

${ }^{8}$ Department of Clinical Medicine, Aarhus University, Aarhus, Denmark

${ }^{9}$ Clinical Trial Services Unit, University of Oxford, Oxford, UK

${ }^{10}$ Institue of Applied Health Research, University of Birmingham Clinical Trials Unit, Birmingham, UK

${ }^{11}$ Birmingham Clinical Trials Unit, University of Birmingham, Birmingham, UK

${ }^{12}$ Department of Radiotherapy, Leiden University Medical Center, Leiden, The

Netherlands

${ }^{13}$ Department of Clinical Oncology, Aarhus University Hospital, Aarhus, Denmark

${ }^{14}$ Department of Pathology, Radboud University Medical Centre, Nijmegen, The

Netherlands

${ }^{15}$ Department of Medical Physics, Aarhus University Hospital, Aarhus, Denmark

${ }^{16}$ Department of Oncology, Odense University Hospital, Odense, Denmark

${ }^{17}$ Department of Medical Oncology, Academic Medical Center, Amsterdam, The Netherlands

${ }^{18}$ Department of Pathology, School of Medicine, University of Leeds, Leeds, UK

${ }^{19}$ Department of Clinical Oncology, Leeds Radiotherapy Research Group, University

of Leeds, Leeds, UK

${ }^{20}$ Department of Clinical Oncology, Leeds Cancer Centre, St James University Hospital, Leeds, UK

Contributors All collaborators made substantial contributions to the design of the study and/or were involved in drafting the manuscript. All collaborators read and approved the final manuscript.

Funding STAR-TREC is an international study, separately funded in each participating country. In the UK, this work was supported by Cancer Research UK (C41557/A19393), in the Netherlands by the Dutch Cancer Society (KWF KUN 2014-7448) and in Denmark by the Danish Cancer Society (R100-A6747). Contact information of Trial sponsor: Birmingham Clinical Trials Unit (BCTU), Institute of Applied Health Research, College of Medical and Dental Sciences, Public Health Building, University of Birmingham, Edgbaston, Birmingham B15 2TT, UK. Phone: 0121415 9104; email: STAR-TREC@trials.bham.ac.uk.

Competing interests None declared.

Ethics approval Commissie mensgebonden onderzoek (CMO).

Provenance and peer review Not commissioned; internally peer reviewed.

Open Access This is an Open Access article distributed in accordance with the terms of the Creative Commons Attribution (CC BY 4.0) license, which permits others to distribute, remix, adapt and build upon this work, for commercial use, provided the original work is properly cited. See: http://creativecommons.org/ licenses/by/4.0/

(c) Article author(s) (or their employer(s) unless otherwise stated in the text of the article) 2017. All rights reserved. No commercial use is permitted unless otherwise expressly granted.

\section{REFERENCES}

1. Atkin WS, Cook CF, Cuzick J, et al. Single flexible sigmoidoscopy screening to prevent colorectal cancer: baseline findings of a UK multicentre randomised trial. Lancet 2002;359:1291-300.

2. UK Colorectal Cancer Screening Pilot Group. Results of the first round of a demonstration pilot of screening for colorectal cancer in the United Kingdom. BMJ 2004;329:133.

3. Bentrem DJ, Okabe S, Wong WD, et al. T1 adenocarcinoma of the rectum: transanal excision or radical surgery? Ann Surg $2005 ; 242: 472-9$. 
4. Peeters KC, Marijnen CA, Nagtegaal ID, et al. The TME trial after a median follow-up of 6 years: increased local control but no survival benefit in irradiated patients with resectable rectal carcinoma. Ann Surg 2007;246:693-701.

5. Endreseth $\mathrm{BH}$, Myrvold HE, Romundstad P, et al. Transanal excision vs. major surgery for T1 rectal cancer. Dis Colon Rectum 2005;48:1380-8.

6. Bökkerink GM, de Graaf EJ, Punt CJ, et al. The CARTS study: Chemoradiation therapy for rectal cancer in the distal rectum followed by organ-sparing transanal endoscopic microsurgery. BMC Surg 2011;11:34

7. Homan J, Bökkerink GM, Aarts MJ, et al. Variation in circumferential resection margin: Reporting and involvement in the SouthNetherlands. Eur J Surg Oncol 2015;41:1485-92.

8. Anderin K, Gustafsson UO, Thorell A, et al. The effect of diverting stoma on long-term morbidity and risk for permanent stoma after low anterior resection for rectal cancer. Eur J Surg Oncol 2016;42:788-93.

9. Kim MJ, Kim YS, Park SC, et al. Risk factors for permanent stoma after rectal cancer surgery with temporary ileostomy. Surgery 2016:159:721-7.

10. Hendren SK, O'Connor BI, Liu M, et al. Prevalence of male and female sexual dysfunction is high following surgery for rectal cancer. Ann Surg 2005;242:212-23.

11. Wallner C, Lange MM, Bonsing BA, et al. Causes of fecal and urinary incontinence after total mesorectal excision for rectal cancer based on cadaveric surgery: a study from the Cooperative Clinical Investigators of the Dutch total mesorectal excision trial. J Clin Oncol 2008;26:4466-72.

12. Engel J, Kerr J, Schlesinger-Raab A, et al. Quality of life in rectal cancer patients: a four-year prospective study. Ann Surg 2003;238:203-13.

13. Temple LK, Bacik J, Savatta SG, et al. The development of a validated instrument to evaluate bowel function after sphincterpreserving surgery for rectal cancer. Dis Colon Rectum 2005;48:1353-65.

14. Lange MM, Marijnen CA, Maas CP, et al. Risk factors for sexual dysfunction after rectal cancer treatment. Eur $J$ Cancer 2009; 45:1578-88

15. Marijnen CA, Kapiteijn E, van de Velde CJ, et al. Acute side effects and complications after short-term preoperative radiotherapy combined with total mesorectal excision in primary rectal cancer: report of a multicenter randomized trial. J Clin Oncol 2002;20:817-25.

16. Grumann MM, Noack EM, Hoffmann IA, et al. Comparison of quality of life in patients undergoing abdominoperineal extirpation or anterior resection for rectal cancer. Ann Surg 2001;233:149-56.

17. Wilson TR, Alexander DJ. Clinical and non-clinical factors influencing postoperative health-related quality of life in patients with colorectal cancer. Br J Surg 2008;95:1408-15.

18. Stephens RJ, Thompson LC, Quirke P, et al. Impact of short-course preoperative radiotherapy for rectal cancer on patients' quality of life: data from the Medical Research Council CR07/National Cancer Institute of Canada Clinical Trials Group C016 randomized clinical trial. J Clin Oncol 2010;28:4233-9.

19. Wiltink LM, Marijnen CA, Meershoek-Klein Kranenbarg E, et al. A comprehensive longitudinal overview of health-related quality of life and symptoms after treatment for rectal cancer in the TME trial. Acta Oncol 2016;55:502-8.

20. Kapiteijn E, Marijnen CA, Nagtegaal ID, et al. Preoperative radiotherapy combined with total mesorectal excision for resectable rectal cancer. N Engl J Med 2001;345:638-46.

21. Tekkis PP, Poloniecki JD, Thompson MR, et al. Operative mortality in colorectal cancer: prospective national study. BMJ 2003;327:1196-201.

22. Bos AC, van Erning FN, Elferink MA, et al. No Difference in Overall Survival Between Hospital Volumes for Patients With Colorectal Cancer in The Netherlands. Dis Colon Rectum 2016;59:943-52.

23. Habr-Gama A, Gama-Rodrigues J, São Julião GP, et al. Local recurrence after complete clinical response and watch and wait in rectal cancer after neoadjuvant chemoradiation: impact of salvage therapy on local disease control. Int J Radiat Oncol Biol Phys 2014;88:822-8.

24. Habr-Gama A, Perez RO, Nadalin W, et al. Operative versus nonoperative treatment for stage 0 distal rectal cancer following chemoradiation therapy. Trans Meet Am Surg Assoc Am Surg Assoc 2004;CXXII:309-16.
25. Maas M, Beets-Tan RG, Lambregts DM, et al. Wait-and-see policy for clinical complete responders after chemoradiation for rectal cancer. J Clin Oncol 2011;29:4633-40.

26. Appelt AL, Pløen J, Harling H, et al. High-dose chemoradiotherapy and watchful waiting for distal rectal cancer: a prospective observational study. Lancet Oncol 2015;16:919-27.

27. Glynne-Jones R, Wallace M, Livingstone JI, et al. Complete clinical response after preoperative chemoradiation in rectal cancer: is a "wait and see" policy justified? Dis Colon Rectum 2008;51:10-20.

28. Renehan AG, Malcomson L, Emsley R, et al. Watch-and-wait approach versus surgical resection after chemoradiotherapy for patients with rectal cancer (the OnCoRe project): a propensity-score matched cohort analysis. Lancet Oncol 2016;17:174-83.

29. Garcia-Aguilar J, Chow OS, Smith DD, et al. Effect of adding mFOLFOX6 after neoadjuvant chemoradiation in locally advanced rectal cancer: a multicentre, phase 2 trial. Lancet Oncol 2015:16:957-66.

30. Bach SP, Hill J, Monson JR, et al. A predictive model for local recurrence after transanal endoscopic microsurgery for rectal cancer. Br J Surg 2009;96:280-90.

31. Cataldo PA, O'Brien S, Osler T. Transanal endoscopic microsurgery: a prospective evaluation of functional results. Dis Colon Rectum 2005;48:1366-71.

32. Restivo A, Zorcolo L, D'Alia G, et al. Risk of complications and long-term functional alterations after local excision of rectal tumors with transanal endoscopic microsurgery (TEM). Int J Colorectal Dis 2016;31:257-66.

33. Kumar AS, Coralic J, Kelleher DC, et al. Complications of transanal endoscopic microsurgery are rare and minor: a single institution's analysis and comparison to existing data. Dis Colon Rectum 2013;56:295-300.

34. You YN, Baxter NN, Stewart A, et al. Is the increasing rate of local excision for stage I rectal cancer in the United States justified?: a nationwide cohort study from the National Cancer Database. Ann Surg 2007;245:726-33.

35. Garcia-Aguilar J, Mellgren A, Sirivongs P, et al. Local excision of rectal cancer without adjuvant therapy: a word of caution. Ann Surg 2000;231:345-51.

36. Ricciardi R, Madoff RD, Rothenberger DA, et al. Population-based analyses of lymph node metastases in colorectal cancer. Clin Gastroenterol Hepatol 2006;4:1522-7.

37. Martens MH, Maas M, Heijnen LA, et al. Long-term Outcome of an Organ Preservation Program After Neoadjuvant Treatment for Rectal Cancer. J Natl Cancer Inst 2016;108:djw171.

38. van Gijn W, Marijnen CA, Nagtegaal ID, et al. Preoperative radiotherapy combined with total mesorectal excision for resectable rectal cancer: 12-year follow-up of the multicentre, randomised controlled TME trial. Lancet Oncol 2011;12:575-82.

39. Lezoche E, Baldarelli M, Lezoche G, et al. Randomized clinical trial of endoluminal locoregional resection versus laparoscopic total mesorectal excision for T2 rectal cancer after neoadjuvant therapy. Br J Surg 2012:99:1211-8

40. Bujko K, Richter P, Smith FM, et al. Preoperative radiotherapy and local excision of rectal cancer with immediate radical re-operation for poor responders: a prospective multicentre study. Radiother Oncol 2013;106:198-205.

41. Verseveld M, de Graaf EJ, Verhoef C, et al. Chemoradiation therapy for rectal cancer in the distal rectum followed by organ-sparing transanal endoscopic microsurgery (CARTS study). Br J Surg 2015:102:853-60.

42. Smart CJ, Korsgen S, Hill J, et al. Multicentre study of short-course radiotherapy and transanal endoscopic microsurgery for early rectal cancer. Br J Surg 2016;103:1069-75.

43. Garcia-Aguilar J, Renfro LA, Chow OS, et al. Organ preservation for clinical T2NO distal rectal cancer using neoadjuvant chemoradiotherapy and local excision (ACOSOG Z6041): results of an open-label, single-arm, multi-institutional, phase 2 trial. Lancet Oncol 2015;16:1537-46.

44. Marijnen CA. Organ preservation in rectal cancer: have all questions been answered? Lancet Oncol 2015;16:e13-e22.

45. Valentini V, Gambacorta MA, Barbaro B, et al. International consensus guidelines on Clinical Target Volume delineation in rectal cancer. Radiother Oncol 2016;120:195-201.

46. Leibold T, Shia J, Ruo L, et al. Prognostic implications of the distribution of lymph node metastases in rectal cancer after neoadjuvant chemoradiotherapy. J Clin Oncol 2008;26:2106-11. 